Jean De Munck, Claude Didry, Isabelle Ferreras, Annette Jobert (eds.), Renewing democratic deliberation in Europe. The challenge of social and civil dialogue

Bruxelles, P.I.E. Peter Lang, coll. « Travail \& Société / Work \& Society », vol. 73, 2012

Laure Bazzoli

\title{
OpenEdition
}

Journals

Édition électronique

URL : http://journals.openedition.org/travailemploi/6523

ISSN : 1775-416X

Éditeur

DARES - Ministère du Travail

Édition imprimée

Date de publication : 1 octobre 2014

Pagination : 74-76

ISSN : 0224-4365

Référence électronique

Laure Bazzoli, « Jean De Munck, Claude Didry, Isabelle Ferreras, Annette Jobert (eds.), Renewing democratic deliberation in Europe. The challenge of social and civil dialogue », Travail et Emploi [En ligne], 140 | octobre-décembre 2014, mis en ligne le 01 octobre 2014, consulté le 22 septembre 2020. URL http://journals.openedition.org/travailemploi/6523

Ce document a été généré automatiquement le 22 septembre 2020

(c) Direction de l'animation de la recherche, des études et des statistiques (Dares) 


\section{Jean De Munck, Claude Didry, Isabelle Ferreras, Annette Jobert (eds.), Renewing democratic deliberation in Europe. The challenge of social and civil dialogue}

Bruxelles, P.I.E. Peter Lang, coll. « Travail \& Société / Work \& Society ", vol. 73, 2012

\section{Laure Bazzoli}

\section{RÉFÉRENCE}

Jean De Munck, Claude Didry, Isabelle Ferreras, Annette Jobert (eds.), Renewing democratic deliberation in Europe. The challenge of social and civil dialogue, Bruxelles, P.I.E. Peter Lang, coll. « Travail \& Société / Work \& Society », vol. 73, 2012, 262 p.

1 Cet ouvrage traite d'un sujet crucial pour notre avenir commun en Europe: la démocratie et son renouveau. Fruit du European Capright project ( $7^{\mathrm{e}}$ programme-cadre financé par la Commission européenne et dirigé par Robert Salais), Renewing democratic deliberation in Europe réunit les contributions de spécialistes européens et américains reconnus venant de plusieurs champs disciplinaires: sociologie, droit et sciences politiques, économie politique, relations industrielles notamment. Rassemblant des connaissances sur les expériences nationales et les transformations en cours, cette recherche ouvre des pistes stimulantes pour analyser le processus démocratique et engager la réforme de ses institutions. "Our book is a beginning", nous disent les éditeurs.

2 Malgré la pluralité des angles d'attaque, cet ouvrage est marqué par une unité de fond. Les auteurs partagent la conviction que la démocratie ne se résume pas à un système 
politique et juridique mais implique également les dimensions sociales et économiques, et que là réside le sens même de l'idée européenne. Ils partagent en outre les référents théoriques de cette conviction: s'inscrivant de manière critique dans la lignée de l'importante littérature sur la notion de démocratie délibérative suscitée par les travaux de Jürgen Habermas et de John Rawls, ils convoquent aussi John Dewey et ils mobilisent en particulier les apports de l'approche par les capacités d'Amartya Sen pour repenser la délibération démocratique dans le contexte européen.

3 Tous les chapitres apportent leur contribution à la thèse principale de l'ouvrage, que l'on peut résumer de la façon suivante. Dans les sociétés postindustrielles, la « question sociale » refait surface notamment sous la forme du problème du déficit démocratique de l'Union européenne. Pour dépasser ce problème majeur, il faut faire évoluer la conception et la pratique de la démocratie en explorant les potentiels d'une démocratie délibérative ancrée dans l'articulation d'un dialogue social renouvelé avec un dialogue civil à élargir et à institutionnaliser. L'enjeu est de réorienter notre modèle de développement économique et de permettre un accomplissement plus abouti de l'idéal démocratique.

Contre l'opposition du social et du civil qui renvoient en fait à deux moments historiques $^{1}$, ce livre entend éviter les positions extrêmes engendrées par la multiplication des discours sur la société civile depuis les années 1990, et qui sont « des formes différentes de la même futilité intellectuelle»(p.13), ainsi que le défend l'introduction générale des éditeurs : d'un côté, un «idéalisme naïf » (ou "idéalisme inconséquent», p.15) qui magnifie la société civile; de l'autre, un "relativisme négatif» (ou «cynisme sans espoir », p. 15) qui récuse tout intérêt à cette notion. Au contraire, les auteurs rendent compte du déploiement de nouvelles formes de participation démocratique et considèrent que c'est l'articulation du civil et du social qui peut régénérer les objectifs et les processus démocratiques. Ce faisant, ils mettent en valeur ce qui nous apparait comme deux apports d'Amartya Sen à cette réflexion : le développement des capacités ("positive freedom») posé comme finalité toujours en devenir de la démocratie ; et l'importance des institutions ou capacités institutionnelles (institutions du dialogue social, droits et «équipement institutionnel» des citoyens, mécanismes de gouvernance) comme "facteurs de conversion » (p. 190) pour étendre la délibération et la participation active des citoyens. L'ouvrage est articulé en quatre parties que l'on peut, comme le suggèrent les éditeurs, regrouper deux par deux. Les deux premières parties analysent les défis que doivent relever les sociétés actuelles pour dépasser les modèles hérités du passé. Les deux dernières délimitent le cadre théorique et pratique d'une réponse à ces défis dans la perspective d'un renouvellement de la démocratie délibérative en Europe.

5 Le premier défi identifié traduit l'objectif majeur de ce renouvellement: nous avons besoin de nouveaux modèles de travail et de prospérité dépassant «les truismes du Fordisme et du Keynésianisme » (p. 14). Ton Korner et Günther Schmid analysent la complexité du "flexibility-security nexus» (p.23) et défendent un modèle négocié incluant dialogue social et civil pour accroître les capacités de transitions soutenables sur le marché du travail, en lien avec une politique macroéconomique et sociale repensée. Dominique Méda retrace le rôle et les difficultés de la société civile dans la défense de nouveaux indicateurs de richesse pour souligner la nécessité d'intégrer les citoyens et les partenaires sociaux à la redéfinition du modèle de croissance. Or, et c'est là le point clé, redéfinir le travail et la prospérité implique un nouveau modèle de 
démocratie économique et sociale: placé au cœur du renouveau démocratique européen par les auteurs de l'ouvrage, cet objectif est exploré spécifiquement par Joel Rodgers. S'appuyant sur l'héritage de la démocratie sociale construite au tournant des $\mathrm{XIX}^{\mathrm{e}}$ et $\mathrm{XX}^{\mathrm{e}}$ siècles - «the one successful egalitarian democratic political experiment under capitalism " (p. 79) -, ce nouveau modèle peut être qualifié de « démocratie productive » car son enjeu est de développer « l'infrastructure productive » de la société (ressources humaines et biens publics). Il repose sur la participation des citoyens à tous les niveaux de l'organisation sociale, en étendant, par une "politique de l'association", l'organisation démocratique au-delà de l'État formel.

6 Le second défi identifié est une conséquence du premier : nous devons faire évoluer les modes de gouvernance du dialogue social, pilier d'une construction européenne solide, pour rendre possibles ces nouveaux modèles de travail et de croissance. Les contributions de Paul Marginson, de Simon Deakin et Aristea Koukiadaki, et de Konstantinos Papadakis se concentrent sur les enjeux de gouvernance démocratique en tirant les leçons des innovations dans le dialogue social à l'heure de la globalisation et dans le contexte transnational de l'Europe. Les innovations étudiées (en particulier le dialogue social européen, les accords cadres internationaux et le dialogue territorial) traduisent, selon ces auteurs, le développement d'une gouvernance à plusieurs niveaux mixant le droit «dur » et le droit «mou », et surtout, d'une forme hybride de dialogue permettant d'intégrer les intérêts multiples de la société civile européenne.

7 La troisième partie constitue le cœur de l'ouvrage car elle en expose le cadre théorique : elle interroge la notion de délibération et défend la nécessité d'articuler, au lieu d'opposer, dialogue social et dialogue civil pour répondre au déficit démocratique de ce début de $\mathrm{xxI}^{\mathrm{e}}$ siècle. Pour Jean De Munck et Isabelle Ferreras, "la distinction analytique » entre négociation (sur les moyens) et délibération (sur les fins) « ne doit pas devenir une distinction ontologique » (p.159). Ces deux auteurs redéfinissent de manière stimulante la notion d'échange démocratique comme articulation de délibération, de négociation et d'expérimentation. La crise actuelle est alors vue comme une dislocation de ces trois composantes du processus démocratique. C'est par leur réarticulation que l'on peut espérer dégager les voies d'un accroissement des capacités de la citoyenneté démocratique. Dans cette perspective, Claude Didry et Annette Jobert soulignent combien le dialogue social est aujourd'hui transformé et ouvre de plus en plus d'espaces de délibération connectant les enjeux du travail et les enjeux de la vie. Serafino Negrelli met en avant, quant à lui, que la démocratie délibérative est une méthode transversale - et non un substitut à la démocratie politique et à la démocratie sociale - qui permet de développer les capacités des citoyens et d'intégrer dans le processus de décision les intérêts affectés par celle-ci. Enfin, Lucio Baccaro, relativisant la portée pratique de la théorie normative de la délibération d'Habermas et Rawls sur la base d'un état des lieux critique des travaux empiriques qu'elle a suscités ${ }^{2}$, propose d'appréhender le dialogue civil comme un postcorporatisme fondé sur une "négociation intégrative ", c'est-à-dire une résolution conjointe de problèmes entre des groupes qui ont des contours plus larges que les intérêts économiques classiques et ne sont pas « single-issue » (pp. 211-212).

8 C'est alors tout logiquement que cet ouvrage se termine en posant la question des nouveaux acteurs et des nouvelles coalitions possibles pour soutenir un tel renouveau de la démocratie délibérative en Europe, sans lesquels les idées ne peuvent trouver à s'inscrire dans le réel. À partir des expériences américaines et européennes, 
Lowell Turner, Béla Galgóczi et Philippe Pochet posent la question essentielle de la façon dont les acteurs historiques de la démocratie sociale peuvent évoluer pour intégrer la société civile et appréhender «le défi de la transformation Verte en Europe » (p. 235). Car l'enjeu, comme cet ouvrage le défend, est bien de traiter en même temps le travail et l'environnement, la vie sociale et la vie humaine pour interroger les fins et non seulement les moyens de la vie économique. Dans cette perspective, reconstruire de manière innovante aussi bien les acteurs collectifs que l'équipement institutionnel d'une démocratie étendue, est une condition d'un nouveau New Deal entre tous les intérêts concernés.

9 Le pari des auteurs est que ce livre ait posé les bases d'une vision renouvelée de la démocratie européenne. Si le défi est relevé, on regrettera cependant que les difficultés de mise en œuvre d'un tel New Deal soient peu traitées, en particulier sa capacité à «discipliner » le pouvoir économique. On aurait également aimé des développements plus directs sur les articulations entre délibération, participation et représentation qui sont au cœur des discussions sur la démocratie délibérative. Un pas important est cependant fait pour "tirer» la notion de délibération dans le sens d'une démocratie radicale que l'on peut qualifier, en suivant le philosophe John Dewey, de "créatrice ", par l'analyse du lien substantiel entre négociation et délibération, par la mise au premier plan de la démocratie économique, et par l'accent sur le caractère profondément expérimental des pratiques et des institutions démocratiques. Les auteurs du présent ouvrage font d'une alliance renouvelée entre dialogue social et dialogue civil la condition de l'avenir du projet européen. Nous les suivons dans cette voie.

\section{NOTES}

1. Au sens où le social émerge à la fin du XIX siècle face au déficit démocratique de la société industrielle, tandis que le civil émerge à la fin $\mathrm{du} \mathrm{xx}^{\mathrm{e}}$ siècle face au déficit démocratique de la société postindustrielle.

2. Pour l'auteur, la recherche empirique qui s'est développée dans les années 2000 pour tester la théorie normative de la délibération est peu concluante : elle offre des résultats contrastés sur les bénéfices sociaux des processus délibératifs et souffre à la fois d'une difficulté d'opérationnalisation des " critères idéalisés » (p. 203) établis par la théorie, et de biais dans les méthodes utilisées (essentiellement «l'approche observationnelle» et «l'approche expérimentale »). 


\section{AUTEURS}

\section{LAURE BAZZOLI}

Université Lyon-2, Triangle UMR 5206 